\title{
Características del sarcoma de Kaposi. Estudio retrospectivo en un hospital de Tercer Nivel
}

\author{
J.A. AVILÉS IZQUIERDO, C. RECARTE GARCÍA-ANDRADE ${ }^{1}$, L. PASTOR \\ GÓMEZ-CORNEJO' ${ }^{1}$, P. LÁZARO OCHAÍTA, J. DE PORTUGAL ÁLVAREZ ${ }^{1}$ \\ Servicio de Dermatología. 'Servicio de Medicina Interna II. Hospital General \\ Universitario Gregorio Marañón. Madrid
}

CHARACTERISTICS OF KAPOSI'S SARCOMA. A RETROSPECTIVE STUDY IN A REFERENCE HOSPITAL

\section{RESUMEN}

El angiosarcoma de Kaposi (SK) es un tumor vascular que afecta a piel y otros órganos. Actualmente se piensa que en su génesis intervienen factores relacionados con la inmunosupresión.

Objetivos: Reflejar los datos correspondientes a la distribución del SK, proporción de subtipos, evolución y terapéutica empleada.

Material y métodos: Estudio retrospectivo de 28 casos. Obtuvimos los datos personales, hábitos, origen del VIH cuando el SK estaba asociado a SIDA, y tratamiento realizado. Tipo de SK y localización, presentación cutánea, complicaciones y síntomas B. Tratamiento realizado, respuesta y efectos indeseables del mismo. Progresión de la enfermedad, tiempo medio de recidiva, supervivencia media y presencia del SK al fallecer.

Resultados: $64 \%$ epidémicos, $21 \%$ tipo clásico y $14 \%$ asociado a trasplantes. En el $48 \%$ de los casos epidémicos, se atribuyó el VIH a hábitos homosexuales y el $18 \%$ a ser ADVP. En todos hubo afectación cutánea, el $46 \%$ tuvo afectación de mucosas, $11 \%$ adenopatías y 14\% extensión visceral. El $71 \%$ recibió tratamiento: $40 \%$ quimioterapia, 35\% radioterapia y $18 \%$ crioterapia. Progresión: el $11 \%$ se estabilizó, en el $50 \%$ hubo extensión local y sistémica en el $17 \%$. La supervivencia media fue de $4,16 \pm 3$ años.

Conclusiones: Hay menor incidencia de SK en la población homosexual. La elevada proporción del clásico y asociado a trasplantes apoya un estado de inmunodepresión como factor predisponente. Hubo numerosas complicaciones locales que se asociaron a síntomas B. La crioterapia se utilizó como primera opción en las formas localizadas y en las generalizadas la quimioterapia.

PALABRAS CLAVE: Angiosarcoma de Kaposi. Epidemiología. VIH. Tratamiento.

\section{ABSTRACT}

Kaposi's angiosarcoma (SK) is a vascular tumour that affects skin and other organs. Nowadays there is thought that immunosuppression is one of the factors related with its genesis.

Objectives: Show the information corresponding to the distribution of the SK, proportion of subtypes, evolution and therapeutic used.

Material and methods: A 28 cases retrospective study. We obtained the personal background, habits, origin of the VIH when the SK was associated with AIDS, and treatment carry out. Type of SK and location, coetaneous presentation, complications and B symptoms. Treatment, response and adverse effects. Progression of the disease, average time of relapse and survival, and presence of the SK at death.

Results: $64 \%$ epidemic, $21 \%$ classic type and $14 \%$ associated with transplants. In $48 \%$ of the epidemic cases, the VIH assumed to homosexual habits and $18 \%$ to being ADVP. In all there was coetaneous affectation, $46 \%$ had affectation of mucouses, $11 \%$ adenophaties and $14 \%$ visceral extension. $71 \%$ received treatment: $40 \%$ chemotherapy, 35\% radiotherapy and $18 \%$ criotherapy. Progression: $11 \%$ became stable, in $50 \%$ there was local extension and systemic in $17 \%$. The average survival was of $4,16 \pm 3$ years.

Conclusions: SK has a minor incidence in the homosexual population. The high proportion of classic and transplants associated affirm the role of inmunosuppression as a predisposing factor. There were numerous local complications that were associated with $B$ symptoms. The criotherapy was used as the first option in the located forms and in the widespread ones was the chemotherapy.

KEY WORDS: Kaposi's angiosarcoma. Epidemiology. HIV. Treatment.

Avilés Izquierdo JA, Recarte García-Andrade C, Pastor Gómez-Cornejo L, Lázaro Ochaíta P, De Portugal Álvarez. J. Caraterísticas del sarcoma de Kaposi. Estudio retrospectivo en un hospital de Tercer Nivel. An Med Interna (Madrid) 2003; 20: 170-174.

\section{INTRODUCCIÓN}

En 1872 Moriz Kaposi (1) describió una neoplasia multifocal, con formación de tumores cutáneos y extracutáneos de origen vascular, a la que denominó "sarcoma pigmentado múltiple idiopático" (2), actualmente Sarcoma de Kaposi (SK), que afectaba principalmente a varones de edad avanzada y ascendencia judía o mediterránea (3). A pesar de que se han descrito casos aislados en ciertas áreas africanas, así como en pacientes yatrogénicamente inmunodeprimidos (4),

Trabajo aceptado: 15 de enero de 2003

Correspondencia: J.A. Avilés Izquierdo. Servicio de Dermatología. Hospital General Universitario Gregorio Marañón. C/. Dr. Esquerdo, 46. 28007 Madrid. 
el SK fue una entidad infrecuente y de escaso interés entre la comunidad médica, hasta que en 1981, la aparición de un elevado número de casos de SK en varones homosexuales en la costa oeste de EE.UU. $(5,6)$ se asoció a la infección por el Virus de Inmunodeficiencia Humana (VIH), siendo ésta la forma más prevalente en la actualidad.

Se han descrito cuatro tipos de SK: clásico; endémico o africano; iatrogénico $(7,8)$; y epidémico o asociado al SIDA. El SK Clásico afecta fundamentalmente a la piel de los miembros inferiores, aunque existen casos de afectación sistémica o visceral. El SK Africano o endémico presenta un amplio espectro clínico, con diferentes manifestaciones y pronóstico según sus distintas variedades (Tabla I). La forma de SK iatrogénico puede cursar con afectación exclusivamente cutánea y remitir espontáneamente al suspender la terapia inmunosupresora, pero también ser muy agresivo con afectación visceral y en ocasiones sin afectación cutánea $(4,9)$. Por último, el SK asociado a SIDA se caracteriza por presentar una distribución multicéntrica y simétrica, y localizarse en zonas presdipuestas (boca, nariz, retroauricular, tronco, pene, piernas y pies). Parece existir una clara relación con el estado inmunitario (menos del 15\% de los pacientes con SK asociado a SIDA tienen más de 500 céls $/ \mathrm{mm}$ de $\mathrm{CD}_{4}$ ). Su progresión es impredecible, aunque generalmente se correlaciona con el estado general del paciente y el nivel de progresión del SIDA. El SK puede ser la primera manifestación de SIDA en el $15 \%$ de los casos.

A nivel cutáneo puede desarrollarse como: Máculas, variables en forma y tamaño, de color rojo-purpúrico, localizadas con mayor frecuencia en pies, paladar duro y punta nasal; Pápulas y Nódulos de 1-2 cms en cuello, tronco y extremidades y Placas purpúricas en zonas distales de las extremidades, con nódulos o formas hiperqueratósicas que semejan psoriasis superficial con fenómeno de Koëbner incluido.

La afectación extracutánea puede ser: Linfática, acompañándose de edema local; Mucocutánea, con lesiones orales en el $45 \%$ de los casos, donde pueden aparecer tanto máculas como pápulas y nódulos. La conjuntiva ocular suele afectarse con frecuencia en forma de máculas relativamente benignas; y Visceral, habitualmente asintomática. Estudios post-mortem sugieren una afectación visceral en más del $75 \%$ de los casos de SK asociados a SIDA, pero en sólo el 10-20\% es la causa de la muerte del paciente. Se localiza con mayor frecuencia en el tracto gastrointestinal y los pulmones, y puede afectar a cualquier órgano (10).

A pesar de su diversidad clínica, estos subtipos de SK comparten unas características anatomopatológicas y fenotípicas comunes que sugieren la intervención de factores genéticos, inmunológicos, endocrinos y microbiológicos en su etiopatogenia. En el SK asociado a SIDA se han ido elaborando diversas líneas de investigación y tratamiento (11-16) para tratar de identificar un probable agente externo cuya transmisión determine el desarrollo del SK, siendo actualmente la asociación más aceptada con dicho sarcoma la infección por virus herpes tipo 8.

El objetivo del presente estudio es reflejar los datos correspondientes a la distribución epidemiológica del SK en nuestro hospital durante un periodo de 7 años, destacando la proporción de cada subtipo respecto al número total de casos, la evolución de estos pacientes y la actitud terapéutica empleada en cada ocasión.

\section{MATERIAL Y MÉTODOS}

Retrospectivamente estudiamos a 44 pacientes diagnosticados inicialmente de "sarcoma de Kaposi" entre los años 1995 y 2001, ambos inclusive. Fueron excluidos los casos que no padecían la enfermedad por estar mal codificados y los que no tenían confirmación anatomopatológica. De los 28 que fueron finalmente incluidos en el estudio, obtuvimos los datos relativos a sus antecedentes personales y epidemiológicos,

\section{TABLA I}

CARACTERÍSTICAS DE LOS DISTINTOS TIPOS DE KAPOSI

\begin{tabular}{llll}
\hline Tipo & Población de riesgo & Edad & Superviviencia \\
\hline Clásico & Europa del Este & $50-80$ & $10-15$ años \\
& Judíos & & (No fallecen por SK) \\
& M editerráneos & &
\end{tabular}

$\begin{array}{ll}\begin{array}{l}\text { Endémico } \\ \text { Benigno nodular } \\ \text { Agresivo } \\ \text { Florido } \\ \text { Asociado a VIH }\end{array} & \begin{array}{l}\text { Africanos de raza negra } \\ \text { Adultos }\end{array} \\ \text { Adenolinfático } & \text { Niños africanos } \\ \text { latrogénico } & \text { Pacientes con enfermedades } \\ \text { (asociado a ID) } & \begin{array}{l}\text { autoinmunes, conectivopatías, } \\ \text { tratados con inmunosupresores } \\ \text { Receptores de trasplantes }\end{array}\end{array}$

2-15 2-3 años

$(x=3)$ 
edad, sexo, hábitos tóxicos y sexuales. En los casos asociados a SIDA reflejamos además el probable mecanismo de transmisión de la infección por VIH, el tiempo transcurrido entre el diagnóstico de la infección por VIH y la aparición del SK, la carga viral y el nivel de linfocitos $\mathrm{CD}_{4}$ al comenzar el SK. También observamos si recibieron tratamiento antirretroviral o profilaxis frente a infecciones oportunistas, si padecieron éstas $\mathrm{u}$ otros procesos tumorales, o si presentaban otras causas de inmunodepresión. Respecto a las características clínicas de la enfermedad, además del tipo de SK y su localización a nivel de piel, mucosas, adenopatías o sistémicas, tuvimos en cuenta la forma cutánea de presentación (máculas, pápulas o nódulos), las complicaciones locales como edemas o úlceras y la presencia o nó de síntomas B. En el apartado terapéutico se ha especificado el tipo de tratamiento realizado, dosis, número de ciclos, intervalo entre ellos, respuesta al tratamiento, los efectos adversos locales y sistémicos del mismo y cuando los pacientes abandonaron voluntariamente el tratamiento, el motivo de dicho abandono. Por último estudiamos la progresión de la enfermedad, el tiempo medio de recidiva, la supervivencia media de los pacientes y la presencia de SK al fallecer.

El análisis estadístico descriptivo se realizó mediante el cálculo de la media y desviación típica de las variables cuantitativas recogidas en el estudio por medio del programa SPSS 9.0.

\section{RESULTADOS}

La distribución entre los distintos tipos de Sarcoma de Kaposi de los 28 casos obtenidos fue la siguiente (Fig. 1): 18 $(64,7 \%)$ asociados a VIH; $6(21,4 \%)$ de tipo clásico, y 4 $(14,3 \%)$ se asociaron a trasplantes de órganos (riñón, hígado y corazón). No se encontró ninguno perteneciente a la forma africana o endémica. La edad media fue de 55 años, con una desviación estándar de 20, debida principalmente a la considerable diferencia de edad entre los casos epidémicos, más jóvenes, y los de tipo clásico (edad media de 86,5 años). La edad de los pacientes estudiados se asoció a una localización diferente de las lesiones, con afectación extracutánea más frecuente en el grupo de población más joven (SK asociados a SIDA) y exclusivamente cutánea en la población de edad avanzada (SK clásico). La mayoría eran varones (23 frente a 5 mujeres) (Tabla II).

Entre los 18 casos de Kaposi epidémicos, en 12 (42\%) se atribuyó el origen del VIH a hábitos homosexuales, en 5 $(18 \%)$ a ser ADVP y en el restante no se pudo filiar el origen

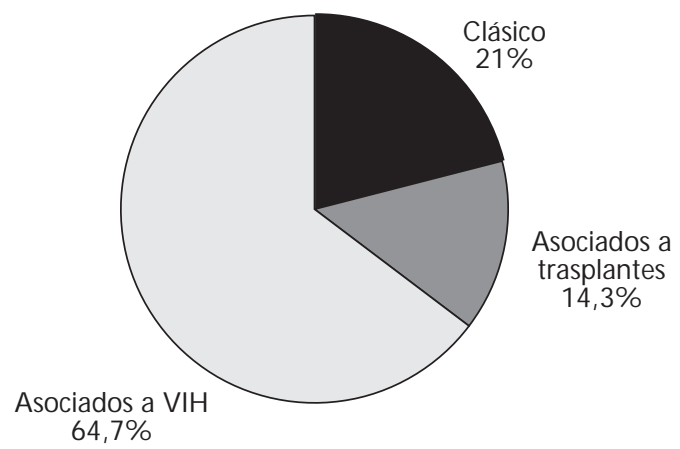

Fig. 1. Subtipos de Sarcoma de Kaposi en nuestra serie.
TABLA II

CARACTERÍSTICAS DE LOS DISTINTO S TIPOS DE SARCO M A DE KAPOSI EN NUESTRO ESTUDIO

\begin{tabular}{lccc}
\hline & Epidémico & Clásico & Yatrogénico \\
\hline Edad media (años) & 43,05 & 86,5 & 61,5 \\
Sexo (V/F) & $17 / 1$ & $3 / 3$ & $3 / 1$ \\
Localización & $\begin{array}{c}\text { Cutáneo-mucosa } \\
\text { Visceral }\end{array}$ & $\begin{array}{c}\text { Cutánea } \\
\text { exclusivamente }\end{array}$ & $\begin{array}{c}\text { Cutáneo- } \\
\text { Mucosa }\end{array}$ \\
\hline
\end{tabular}

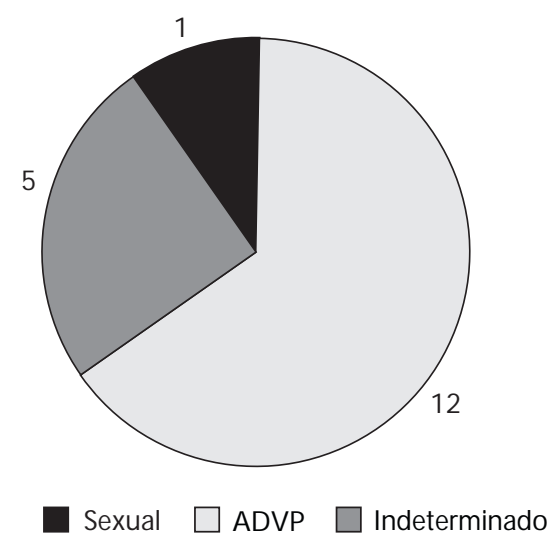

Fig. 2. O rigen del VIH en el Kaposi Epidémico.

(Fig. 2). El tiempo medio entre la infección por el VIH y el diagnóstico del sarcoma de Kaposi fue de 2,09 $\pm 3,5$ años. El valor medio de $\mathrm{CD}_{4}$ al inicio de la enfermedad fue de 154,63 céls $/ \mathrm{mm}^{3}$, y el de la carga viral de 32.604 copias $/ \mu 1$.

En los 28 pacientes hubo afectación cutánea, existiendo además lesiones en mucosas, principalmente oral, en 13 $(46,4 \%)$, adenopatías presumiblemente atribuibles al sarcoma en $3(11 \%)$ y extensión visceral -aparato digestivo y respiratorio- en 4 (14,3\%) (Fig. 3).

Las lesiones adoptaron formas exclusivamente maculares en el $8 \%$; únicamente papulosas en el $36 \%$ y nodulares en el $56 \%$.

Como complicaciones locales el 42,9\% desarrolló edemas y el $10,7 \%$ úlceras. El $32 \%$ de los afectados sufrió síntomas B en principio debidos al sarcoma de Kaposi (Tabla III).

Con respecto al tratamiento, el $71,4 \%$ de los pacientes recibió tratamiento específico frente al SK. La terapia más utilizada fueron diferentes pautas de mono y poliquimioterapia $(39,3 \%)$, siendo la daunorrubicina el citostático más empleado. El $35,7 \%$ recibió radioterapia, y un $17,9 \%$ fue tratado con crioterapia local. Solamente en dos casos se empleó el Interferon sistémico, con mal resultado (Fig. 4). Se describen efectos adversos secundarios al tratamiento en el $38 \%$ de los pacientes, abandonando el mismo de forma voluntaria 5 de ellos.

La evolución de la enfermedad en nuestra serie varió desde la estabilización en el $11 \%$ de los pacientes, hasta exten- 


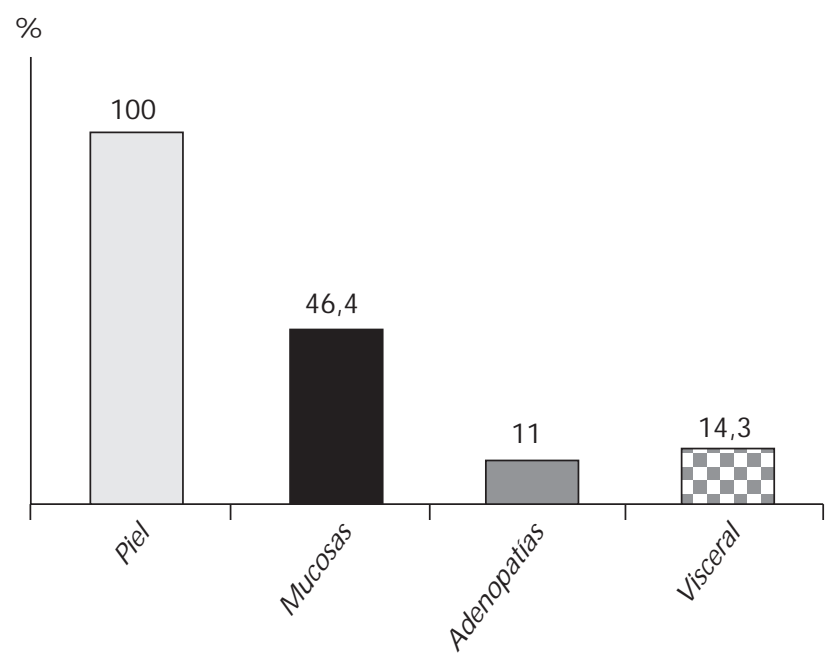

Fig. 3. Afectación proporcional en las diversas localizaciones del organismo

\section{TABLA III}

\section{PO RCENTAJE DE COM PLICACIONES LO CALES Y} SISTEM ICAS

\section{Síntomas B}

\begin{tabular}{lll} 
Complicaciones & Úlceras & $10,7 \%$ \\
locales & Edemas & $42,9 \%$ \\
\hline
\end{tabular}

sión local del sarcoma en el 50\% y progresión sistémica en el $17 \%$ de los casos figura 5). El tiempo medio de recidiva fue aproximadamente de un año. La supervivencia media estimada fue de 4,16 $\pm 3,15$ años, encontrándose una asociación entre el tiempo medio de recidiva, la supervivencia media y la remisión después del tratamiento (a mayor tiempo de recidiva, mayor supervivencia y mayor probabilidad de remisión). Diez pacientes habían fallecido al realizar el presente estudio, aunque no se llegó a establecer ningún tipo de relación entre el sarcoma de Kaposi y la causa del éxitus.

\section{DISCUSIÓN}

Cuando se comenzó a estudiar la epidemiología del sarcoma de Kaposi hace 20 años, la mayoría de las series reflejaban una prevalencia del 95\% de SK asociado a VIH en homosexuales, siendo muy escasos los pacientes con SK asociado a VIH no homosexuales (17). En nuestra serie, existe una menor proporción de casos de SK en la población homosexual (42\% del total, $64,7 \%$ de los casos asociados a SIDA), debida probablemente a una mayor concienciación de dicha población frente al SIDA y a una mayor utilización de medidas protectoras ante las enfermedades de transmisión sexual.

La significativa proporción de casos de SK clásico $(21,4 \%)$ y los asociados a inmunosupresión iatrogénica
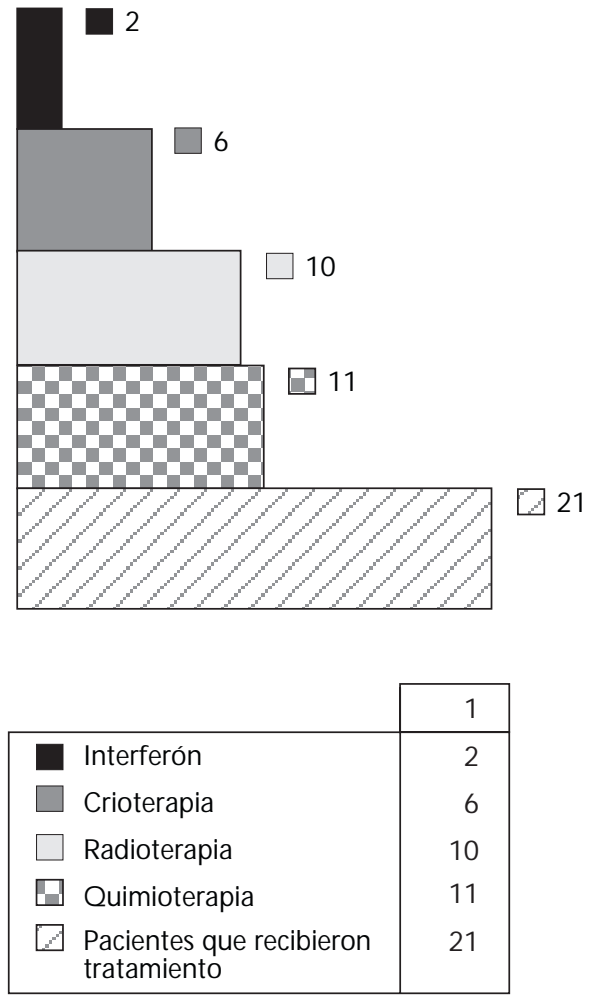

Fig. 4. Tratamiento utilizado.

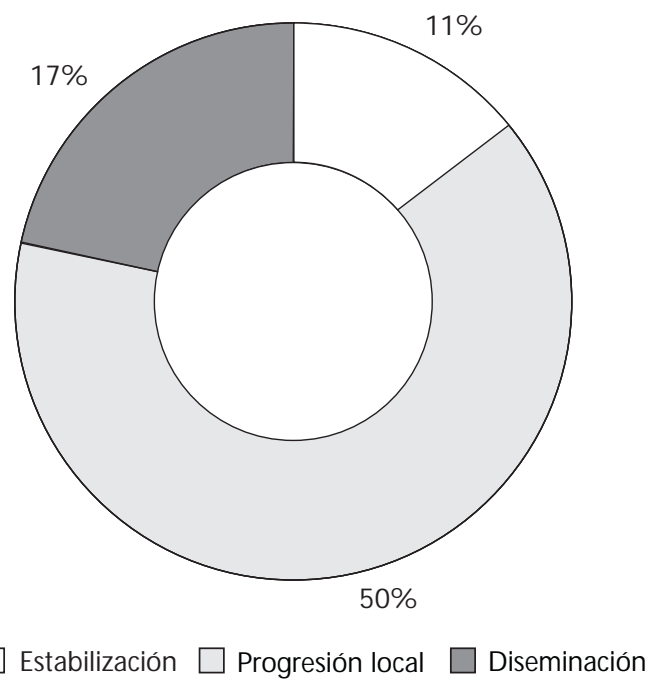

Fig. 5. Evolución del Sarcoma.

$(14,3 \%)$, apoyan las teorías sobre la necesidad de un estado de inmunodepresión para el desarrollo del SK, en relación con la edad avanzada y pluripatologías crónicas en el primer grupo y los tratamientos inmumosupresores postrasplante en el segundo, por lo que dada la tendencia a una mayor longevidad media de la población y a un uso cada vez más frecuente de terapias inmunosupresoras, no se puede menospreciar su existencia, ni asociar de forma exclusiva el SK y el SIDA. 
Con respecto a la distribución y extensión de las lesiones -afectación cutánea, mucosas, adenopatías y vísceral- destacar que coincide con las de otras series de SK realizadas en nuestra comunidad (18). Solamente el $8 \%$ presentaban afectación cutánea en forma de máculas, desarrollando el resto lesiones papulosas y nódulos. Un importante número de pacientes sufrió complicaciones locales, con edemas y úlce-

\section{Bibliografía}

1. Fitzpatrick TB. Dermatology in general medicine. 5th ed. New York: Mc Graw-Hill; 1999: p. 1195.

2. Lázaro Ochaita P. Dermatología: texto y atlas. $2^{\mathrm{a}}$ ed. Madrid.: Meditécnica SA; 1993: p. 542.

3. Brenner B, Weissman-Brenner A, Rakowsky E, Weltfriend S, Fenig E, Friedman-Birnbaum R, et al. Classical Kapossi Sarcoma: Pronostic factor analysis of 248 patients. Cancer 2002 Nov 1; 95 (9): 1982-7.

4. Bahat E, Akman S, Karpuzoglu G, Atkan S, Ucar T, Arslan AG, et al: Visceral Kaposi's sarcoma with intracranial metastasis: A rare complication of renal transplantation. Pediatr Transplant 2002 Dec. 6 (6): 505-8.

5. Centers for Disease Control. Kaposi's sarcoma and Pneumocystis carinii pneumonia among homosexual men-New York City and California. MMWR 1981 Jul 3; 30 (25): 305-8.

6. Centers for Disease Control. Special Report: Epidemiologic aspects of the current outbreak of Kaposi's sarcoma and opportunistic infections. N Engl J Med 1982 Jan 28; 306 (4): 248-52.

7. Trattaner A, Hodak E, David M, Sandbank M. The appearance of Kaposi sarcoma during corticosteroid therapy. Cancer 1993; 72 (5): 1779-83.

8. Reizis Z, Trattner A, Katzenelson V, David M, Rotem A, Nativ O, et al. Flow cytometric DNA analysis of classic and steroid-induced Kaposi $\pm \mathrm{s}$ sarcoma. Br J Dermatol 1995; Apr 132 (4): 548-50.

9. Tappero JW, Conant MA, Wolfe SF, Berger TG. Kaposi \pm s sarcoma: Epidemiology, pathogenesis, histology, clinical spectrum, staging criteria and therapy. J Am Acad Derm 1993 Mar 28; (3): 371-87.

10. Miras Parra FJ, Muñoz Medina L, Álvarez de Cienfuegos Rodríguez A, Gómez Jiménez FJ, Gazquez Pérez I. Afectación multisistémica en el ras, que se asociaron a la existencia de sintomatología sistémica (fiebre, astenia, pérdida de peso).

También es significativa la relación entre el número de lesiones y la terapia empleada. En las formas localizadas se utilizó la crioterapia con Nitrógeno líquido como primera opción, y las formas generalizadas se trataron mayoritariamente con quimioterapia. sarcoma de Kaposi clásico agresivo. An Med Interna (Madrid) 2002; 19: 212-3.

11. Huang YQ, Li JJ, Rush MG, Poiesz BJ, Nicolaides A, Jacobson M, et al. HPV-16 related DNA sequences in Kaposits sarcoma. Lancet 1992 feb 29; 339 (8792): 515-8.

12. Chang Y, Cesarman E, Pessin MS, Lee F, Culpepper J, Knowles DM, et al. Identification of herpesvirus-like sequences in AIDS-associated Kaposi \pm s sarcomas. Science 1994 dec 16; 266 (5192): 1865-9.

13. Ensoli B, Gendelman R, Markham P, Fiorelli V, Colombini S, Raffeld $\mathrm{M}$, et al. Synergy between basic fibroblast growth factor and HIV-1 Tat protein in induction of Kaposi=s sarcoma. Nature 1994 oct 20; 371(6499): 674-80.

14. Moore PS, Gao SJ, Domínguez G, Cesarman E, Lungu O, Knowles DM, et al. Primary characterization of a herpesvirus agent associated with Kaposi=s sarcoma. J Virol 1996; 70: 549-58.

15. Gill J, Bourboulia D, Wilkinson J, Hayes P, Cope A, Marcelin AG. et al. Prospective study of the effects of antiretroviral therapy on Kaposi sarcoma-associated herpesvirus infection in patients with and without Kaposi sarcoma. J Acquir Inmune Defic Syndr 2002 Dec 1; 31(4): $384-90$.

16. Ablashi DV, Chatlynne LG, Whitman JE Jr, Cesarman E. Spectrum of Kaposi's sarcoma -associated herpesvirus, or human herpesvirus 8 , diseases. Clin Microbiol Rev 2002 Jul; 15(3): 439-64.

17. Masood R, Cai J, Law R, Gill P. AIDS associated Kaposi \pm s sarcoma: pathogenesis, clinical features and treatment. Curr Opin Oncol 1993; 5(5): 831-4.

18. Rubio García R. Tumores asociados al sida. Medicine (Madrid) 1998; 85: 3983-6. 